\title{
Dictynna
}

Dictynna

Revue de poétique latine

11 | 2014

Varia

\section{Capri e porci: Priap. 65 e i suoi modelli ellenistico- romani}

Enrico Magnelli

\section{Q OpenEdition}

Journals

\section{Edizione digitale}

URL: http://journals.openedition.org/dictynna/1035

DOI: 10.4000/dictynna.1035

ISSN: 1765-3142

\section{Notizia bibliografica digitale}

Enrico Magnelli, «Capri e porci: Priap. 65 e i suoi modelli ellenistico-romani », Dictynna [En ligne], 11 2014, mis en ligne le 25 novembre 2014, consulté le 10 septembre 2020. URL : http:// journals.openedition.org/dictynna/1035; DOI : https://doi.org/10.4000/dictynna.1035

Questo documento è stato generato automaticamente il 10 settembre 2020.

\section{(c) (i) (9)}

Les contenus des la revue Dictynna sont mis à disposition selon les termes de la Licence Creative Commons Attribution - Pas d'Utilisation Commerciale - Pas de Modification 4.0 International. 


\title{
Capri e porci: Priap. 65 e i suoi modelli ellenistico-romani
}

\author{
Enrico Magnelli
}

Hic tibi, qui rostro crescentia lilia mersit, caeditur e tepida victima porcus hara: ne tamen exanimum reddas pecus omne, Priape, horti sit facias ianua clausa tui.

1 mersit codd. fere omnes: morsit $\mathbf{X}^{1}$, coniecerat Bücheler : carpsit vel rasit vel rosit Heinsius 3 ne $\mathbf{X}^{2}$ : nec cett. exanimum Laur. 33.22 : extrarium $\mathbf{V}$ : extraneum cett. : extinctum ed. Ald. : externum Clairmont : esca meum Kloss : exta meum Gärtner reddas Gärtner : facias codd.: fiat Kloss 4 facias codd. : posthac Bücheler ${ }^{1}$

2 Sul carme 65 del Corpus Priapeorum si è discusso non poco in tempi recenti. Per quanto riguarda la constitutio textus, se sul primo distico gli studiosi sono ormai concordi (sulla lezione mersit avremo modo di tornare), lo stesso non può dirsi del secondo, ove la ripetizione facias... facias è sospetta ${ }^{2}$ : si è cercato di giustificarla vedendovi «a play on the use of the same word for two different syntactic functions» ${ }^{3}$, ma credo che abbiano ragione Gerrit Kloss e Thomas Gärtner ${ }^{4}$ a mantenere il secondo facias - che ricalca le costruzioni di fac o di faciant + congiuntivo tipiche del linguaggio delle preghiere ${ }^{5}-$ individuando invece il guasto nel v. 3. Sono tutt'altro che rari i casi di corruttele verificatesi non perché lo scriba avesse ancora in mente ciò che aveva trascritto poco prima, bensì perché i suoi occhi correvano a ciò che nel suo modello figurava poco dopo $^{6}$ : qui reddas di Gärtner ${ }^{7}$ è probabilmente la soluzione, anche se non lo seguirei nel correggere il vagamente patetico exanimum in exta meum. Minore attenzione l'epigramma ha ricevuto dal punto di vista esegetico e storico-letterario. Ad un primo livello di lettura, si tratta solo di un bonario, ironico ammonimento al dio degli orti affinché non si ripeta la necessità di sacrificargli altri animali (le ipotesi che vi scorgono allusioni sessuali di varia natura - porcus come xoĩ pos/cunnus, o altro - mi sembrano francamente poco verosimili8). Rigettando il severo giudizio di Hermann Tränkle, che lo definiva «nichts weiter als eine matte, absurd übertreibende Witzelei, auch in der Formulierung von bescheidener Qualität» ${ }^{9}$, Kloss e Gärtner vi vedono piuttosto una maliziosa allusione all'avidità di Priapo, che con questo sistema, se lo si lascia fare, 
potrà procurarsi molte altre vittime ${ }^{10}$; secondo Callebat, da un punto di vista più generale «le carmen 65 propose une variante originale du thème de la dédicace, à la fois par l'anecdote pittoresque qui motive l'offrande, par la simplicité du cadre paysan et par la légèreté ironique attachée à l'évocation implicite tant du dédicataire (gardien négligent) que du dédicant, socieux de conserver son troupeau plus que d'honorer le dieu. C'est dans ce tableau faussement naïf que doit être identifié l'intérêt essentiel de cette priapée» ${ }^{11}$.

3 C'è anche dell'altro. Nessuno, a quanto mi risulta, si è mai soffermato sui possibili modelli ellenistici di questo carme, eppure io credo che in un lettore colto del I o II sec. d.C. ${ }^{12}$ il tema del primo distico - l'animale che danneggia le piante protette da un dio e viene perciò sacrificato a quest'ultimo - non potesse non evocare precedenti letterari di una certa importanza. Nella tradizione greca era il capro a fare una brutta fine: l'esempio più noto è un epigramma di Leonida di Taranto, AP 9, 99 = HE 2161-6:

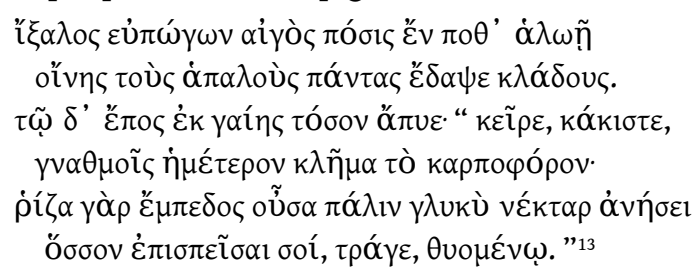

Leonida è imitato, a quanto sembrerebbe, da Eveno (Ascalonita?), AP 9, $75=$ GPh 2308-9,

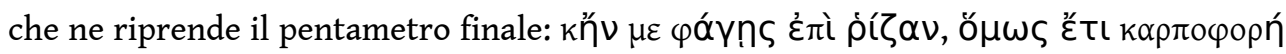

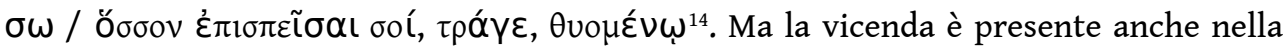
tradizione esopica, fab. 327 Hausrath-Hunger 339 Chambry ${ }^{15}$; e una situazione affine è

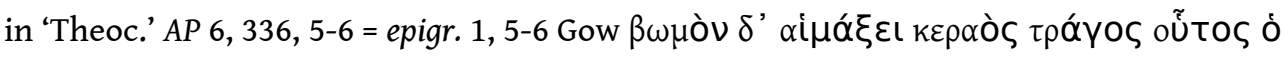

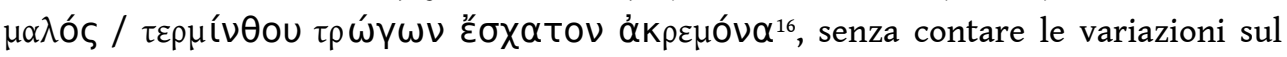
tema del 'sacrificio come contrappasso' ad opera di anon. AP 6, $45=$ HE 3842-5 e 6, $169=$ FGE 1120-3 (il riccio) e di Phil. AP 11, 33 = GPh 3023-6 (l'edera), nonché, molto più tardi, di Agath. AP 6, $72=67$ Viansino (la lepre) ${ }^{17}$. Non meno celebre di Leonida era poi l' Erigone di Eratostene, in cui parimenti si narrava - con fini verosimilmente eziologici, forse anche in relazione alle teorie sull'origine della tragedia - il sacrificio di un capro che aveva osato divorare le foglie della vite da poco scoperta ${ }^{18}$. La tradizione prosegue nella poesia augustea, e se Virgilio riprende il motivo usuale in georg. 2, 380-1 (non aliam ob culpam Baccho caper omnibus aris / caeditur), è Ovidio a fornire il precedente più vicino al nostro carme priapeo, abbinando l'uccisione espiatrice del capro con quella del maiale: questo sia nelle Metamorfosi $(15,111-115)$,

$$
\text { et prima putatur }
$$

hostia sus meruisse mori, quia semina pando eruerit rostro spemque interceperit anni; vite caper morsa Bacchi mactandus ad aras ducitur ultoris. nocuit sua culpa duobus,

sia nei Fasti $(1,349-361)$, in cui si precisa che la morte della scrofa è voluta da Cerere, ulta suas merita caede nocentis opes (v. 350), e si offre una versione latina, quasi una traduzione, delle minacce della vite al capro (rode, caper, vitem; tamen hinc, cum stabis ad aram, / in tua quod spargi cornua possit erit, vv. 357-358) ${ }^{19}$. Non guasta notare che il v. 2 del nostro carme priapeo esibisce una vistosa eco ovidiana, da am. 3, 13, 16 et minor ex humili victima porcus hara (come hanno ben sottolineato i commentatori dell'uno e dell'altro testo). 
6 È questo retroterra che, a mio avviso, rendeva capace il lettore colto di apprezzare in pieno l'ironico scarto di Priap. 65 rispetto alla tradizione letteraria precedente. Il primo distico ha un tono volutamente solenne, adatto a rievocare la serietà dei modelli ellenistici ed ovidiani - un motivo in più, sebbene non ve ne fosse bisogno, per non correggere mersit al v. 1 in morsit, semanticamente più scialbo ("ha morso" invece del molto migliore "ha devastato") e morfologicamente implausibile ${ }^{20}$. Nel secondo distico si cambia tono, riconducendo il tutto a una dimensione molto pratica e concreta, adatta non a Dioniso o a Cerere bensì al modesto Priapo (cfr. 14, 5-7 istuc caelitibus datur severis: / nos vappae sumus et pusilla culti / ruris numina). Lungi dal fornire l'eziologia di culti attici e magari della tragedia, come in Eratostene, o dal segnare l'inizio dell'epoca delle vittime cruente, come in Ovidio, il sacrificio del porcello diviene qui un incidente di percorso: quelli erano l'atto fondativo di una tradizione, questo invece, nell'umile prospettiva dell'offerente, non dovrà ripetersi ("prevenire è meglio che curare"). Del resto, al lettore che avesse in mente i modelli ovidiani non sarà sfuggito come, tra i due animali ivi menzionati, l'avido ed astuto dio degli orti abbia diretto le sue mire non sul capro bensì sul più appetitoso suino. Ancora una volta, i Carmina Priapea si divertono a reinterpretare il patrimonio letterario del passato in modo scherzosamente irriverente.

\section{BIBLIOGRAFIA}

D. Accorinti, Nonno di Panopoli. Le Dionisiache, IV (canti XL-XLVIII), Milano 2004

M.G. Albiani, Il capro e il terebinto (Theocr. AP VI 336 = Ep. I Gow), «Eikasmós» 7, 1996, pp. 161-163

B. Bergmann, A Painted Garland: Weaving Words and Images in the House of the Epigrams in Pompeii, in Z. Newby - R. Leader-Newby (eds.), Art and Inscriptions in the Ancient World, Cambridge 2007, pp. 60-101

E. Bianchini, Carmina Priapea, Milano 2001

V. Buchheit, Studien zum Corpus Priapeorum, München 1962

V. Buchheit, Einheit und Zeit der Carmina Priapea, «Hermes» 135, 2007, pp. 74-79

F. Bücheler, Vindiciae libri Priapeorum, «RhM» 18, 1863, pp. 381-415 = Id., Kleine Schriften I, LeipzigBerlin 1915, pp. 328-362

L. Callebat, Priapées, avec une étude métrique par J. Soubiran, Paris 2012

I. Cazzaniga, Carmina ludicra Romanorum: Pervigilium Veneris - Priapea, Augustae Taurinorum 1959

M. Citroni, Les proèmes des Priapées et le problème de la datation du recueil, in F. Biville - E. Plantade -

D. Vallat (eds.), « Les vers du plus nul des poètes... ». Nouvelle recherches sur les Priapées, Lyon 2008, pp. 35-51

R.E. Clairmont, Carmina Priapea, diss. Chicago 1983

C. De Stefani, Cruces dell'Inno a Delo (vv. 1; 205; 249), «Maia» 49, 1997, pp. 93-98 
T. Gärtner, Untersuchungen zur Einheit und Textgestalt der Priapeen. Das lateinische Priapeen-Corpus im Kontext antiker Priap-Dichtung, «GFA» 10, 2007, pp. 147-250

J. Geiger, Euenus of Ascalon, «SCI» 11, 1991-92, pp. 114-122

C. Goldberg, Carmina Priapea. Einleitung, Übersetzung, Interpretation und Kommentar, Heidelberg 1992

R. Höschele, Die blütenlesende Muse. Poetik und Textualität antiker Epigrammsammlungen, Tübingen 2010

A.E. Housman, Mergere and Priap. 65, «CR» 29, 1915, pp. 173-174 = Classical Papers III, Cambridge 1972, pp. 915-916

H.M. Jackson - C.E. Murgia, Notes on Problems in the Text of «Carmina Priapea», «MD» 37, 1996, pp. $245-270$

G. Kloss, Überlegungen zur Verfasserschaft und Datierung der Carmina Priapea, «Hermes» 131, 2003, pp. 464-487

E. Livrea, Nonnus and the Orphic Argonautica, in K. Spanoudakis (ed.), Nonnus of Panopolis in Context. Poetry and Cultural Milieu in Late Antiquity, Berlin-Boston 2014, pp. 55-76

J. Moore-Blunt, Enemies of the Vine. Three Epigrams, «Mnemosyne» n.s. 34, 1981, pp. 322-330

E.M. O'Connor, Dominant Themes in Greco-Roman Priapic Poetry, diss. Santa Barbara 1984

E.M. O'Connor, Symbolum Salacitatis. A Study of the God Priapus as a Literary Character, Frankfurt am Main 1989

N. Piacenza, La vigna di Leonida e il capro di Eronda: favola e critica nell'epigramma 9,99 dell'Anthologia Palatina, «ARF» 10, 2008, pp. 33-37

É. Prioux, Petits musées en vers: épigramme et discours sur les collections antiques, Paris 2008

A. Rosokoki, Die Erigone des Eratosthenes, Heidelberg 1995

L. Rossi, The Epigrams Ascribed to Theocritus: A Method of Approach, Leuven-Paris-Sterling, VA 2001

H. Tränkle, Entstehungszeit und Verfasserschaft des Corpus Priapeorum, «ZPE»124, 1999, pp. 145-156

D. Vallat, Martial et les Priapées: l'angle onomastique, «Maia» 58, 2006, pp. 33-45

F. Williams, Aphrodite’s Secret Weapon (Colluth. Rapt. Hel. 95), «Eikasmós» 12, 2001, pp. 179-183

\section{NOTE}

1. Offro qui un apparato critico semplificato ai fini della presente discussione. Per le lezioni dei manoscritti mi baso primariamente sul recente e valido L. Callebat, Priapées, Paris 2012 (ormai l'edizione critica di riferimento, di cui adotto i sigla), p. 27, integrato con l'amplissimo, seppur a volte impreciso, R.E. Clairmont, Carmina Priapea, diss. Chicago 1983, pp. 311-313, e con le emendazioni proposte nei lavori di Kloss e di Gärtner (citati infra).

2. «Probabile non est sine ulla necessitate gratiave facias verbum in hoc versu poetam iterasse; immo respicientem superiora librarium repetitionem illam commisisse puto» (F. Bücheler, Vindiciae libri Priapeorum, «RhM» 18, 1863, p. 405 = Id., Kleine Schriften I, Leipzig-Berlin 1915, p. 352).

3. «The redoubling of facias, far from being without reason, adds a touch of irony to the proposed alternative («Lest you make ..., do you make...»)»: così Jackson in H.M. Jackson - C.E. 
Murgia, Notes on Problems in the Text of "Carmina Priapea», «MD»37, 1996, p. 259, seguito da Callebat, op. cit., p. 264.

4. G. Kloss, Überlegungen zur Verfasserschaft und Datierung der Carmina Priapea, «Hermes»131, 2003, p. 479; T. Gärtner, Untersuchungen zur Einheit und Textgestalt der Priapeen. Das lateinische PriapeenCorpus im Kontext antiker Priap-Dichtung, «GFA»10, 2007, pp. 224-225.

5. Come ha ben visto C. Goldberg, Carmina Priapea. Einleitung, Übersetzung, Interpretation und Kommentar, Heidelberg 1992, p. 317, con bibliografia e paralleli (tra cui Priap. 42, 4 fac veros fructus ille, Priape, ferat). La correzione in posthac di Bücheler era guardata con favore da I. Cazzaniga, Carmina ludicra Romanorum: Pervigilium Veneris - Priapea, Augustae Taurinorum 1959, p. 45.

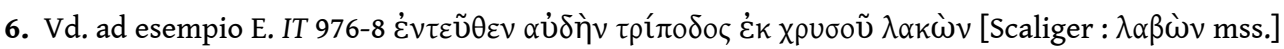

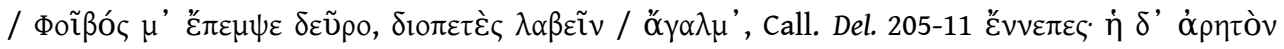

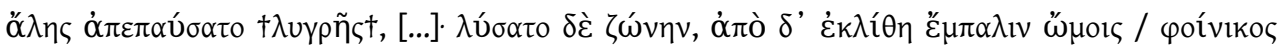

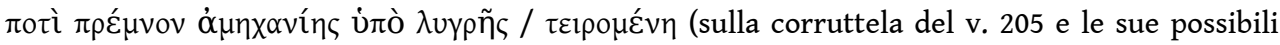
soluzioni cfr. C. De Stefani, Cruces dell'Inno a Delo (vv. 1; 205; 249), «Maia» 49, 1997, pp. 95-96), Posidipp. AP 12, 168, 1-3 (HE 3086-8 = 140, 1-3 A.-B.) Navvoũ

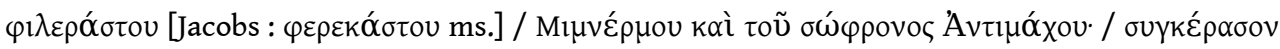

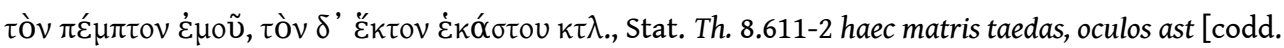
plerique : contra Poynton : gemit P] illa paternos, / altera regnantem, profugum gemit altera fratrem,

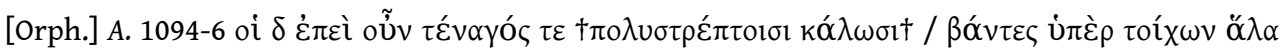

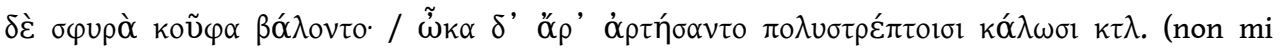
convince la difesa del testo tràdito ad opera di E. Livrea, Nonnus and the Orphic Argonautica, in K. Spanoudakis [ed.], Nonnus of Panopolis in Context. Poetry and Cultural Milieu in Late Antiquity, Berlin-

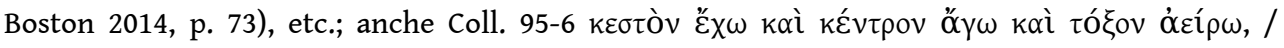

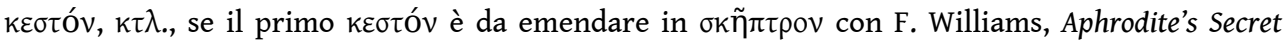
Weapon (Colluth. Rapt. Hel. 95), «Eikasmós» 12, 2001, pp. 179-183.

7. Uso di reddere del tutto normale (cfr. OLD s.v. reddo, 17); Gärtner, art. cit., p. 225, cita giustamente anche Priap. 47, 4.

8. Esempi in E.M. O'Connor, Dominant Themes in Greco-Roman Priapic Poetry, diss. Santa Barbara 1984, pp. 347-348, ripreso in Id., Symbolum Salacitatis. A Study of the God Priapus as a Literary Character, Frankfurt am Main 1989, p. 151; E. Bianchini, Carmina Priapea, Milano 2001, pp. 298-299.

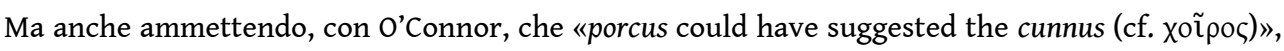
l'idea che «hara (v. 2) is a possible metaphor for the vagina or anus (cf. CP 73. 4)» è incompatibile col concetto di 'provenienza' (e tepida... porcus hara), e manca di paralleli (certo non Priap. 73, 4: hara ed ara non sono la stessa cosa). Giustamente Callebat, op. cit., p. 262 osserva che «ce type d'interprétation repose en réalité sur l'a priori discutable du caractère uniformément obscène des différentes pièces du recueil».

9. H. Tränkle, Entstehungszeit und Verfasserschaft des Corpus Priapeorum, «ZPE» 124, 1999, p. 154.

10. Kloss, art. cit., p. 479; Gärtner, art. cit., pp. 153-154. Non dissimile - come mi fa opportunamente notare un anonimo referee, che qui ringrazio - il caso di Priap. 77: il dio ha tutto l'interesse a lasciare accessibile il giardino, così che i ladri vengano e lui possa punirli nel modo che tanto gli piace (per un'esegesi poetologica di quest'ultimo carme vd. ora R. Höschele, Die blütenlesende Muse. Poetik und Textualität antiker Epigrammsammlungen, Tübingen 2010, pp. 299-300).

11. Callebat, op. cit., p. 262. Cfr. V. Buchheit, Studien zum Corpus Priapeorum, München 1962, p. 77 («c. 65 kontaminiert ebenfalls in kürzester Form mehrere in sich unabhängige Motive»). Osservazioni interessanti sulla relazione tra questi versi e il carme 70 (in cui viceversa è Priapo a raccomandarsi, tra il serio e il faceto, che animali ingordi non siano lasciati avvicinarsi a lui) offre Gärtner, art. cit., p. 230. 
12. Sulla datazione (tuttora assai discussa) dei Priapea vd. i già citati studi di Tränkle e di Kloss, nonché D. Vallat, Martial et les Priapées: l'angle onomastique, «Maia» 58, 2006, pp. 33-45, e V. Buchheit, Einheit und Zeit der Carmina Priapea, «Hermes» 135, 2007, pp. 74-79. Ovviamente la data di singoli carmi può non coincidere con quella dell'allestimento della raccolta: utili in tal senso le precisazioni di M. Citroni, Les proèmes des Priapées et le problème de la datation du recueil, in $\mathrm{F}$. Biville - E. Plantade - D. Vallat (eds.), «Les vers du plus nul des poètes... ». Nouvelle recherches sur les Priapées, Lyon 2008, pp. 35-51.

13. Rimango piuttosto scettico sulla possibilità di leggere questo epigramma in chiave metaletteraria, secondo l'ipotesi peraltro interessante di N. Piacenza, La vigna di Leonida e il capro di Eronda: favola e critica nell'epigramma 9,99 dell'Anthologia Palatina, «ARF»10, 2008, pp. 33-37. Il testo non sembra fornire indizi, anche velati, in tal senso.

14. Un distico di per sé alquanto laconico, se non era affiancato da una didascalia o da una raffigurazione della scena: significativo al riguardo il ritrovamento a Pompei di un affresco che rappresenta un capro intento a mordere una vite, corredato giustappunto del nostro epigramma (Ep. Gr. 1106 Kaibel: vd. in proposito B. Bergmann, A Painted Garland: Weaving Words and Images in the House of the Epigrams in Pompeii, in Z. Newby - R. Leader-Newby [eds.], Art and Inscriptions in the Ancient World, Cambridge 2007, pp. 60-101; É. Prioux, Petits musées en vers: épigramme et discours sur les collections antiques, Paris 2008, pp. 29-63). Sulla paternità dell'epigramma vd. soprattutto J. Geiger, Euenus of Ascalon, «SCI» 11, 1991-92, pp. 116-118. Gow e Page, GPh II p. 291, vedono invece in Leonida l'imitatore («we think it very probabile that the couplet is a relatively old one, known to Leonidas, who adopted its second line for a context of his own»); la loro opinione è seguita da M.G. Albiani, Il capro e il terebinto (Theocr. AP VI 336 = Ep. I Gow), «Eikasmós» 7, 1996, pp. 161-162. Vale la pena di notare che un altro epigramma attribuito ad uno degli 'Eveni' della Anthologia,

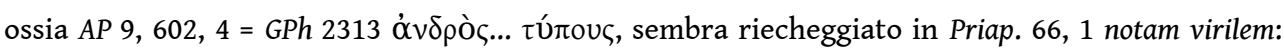
cfr. Gow e Page, GPh II p. 292, Goldberg, op. cit., p. 317, e Callebat, op. cit., p. 264 (ringrazio un anonimo referee per aver richiamato la mia attenzione su questo).

15. Da cui 'Babr.' 181 Crusius, secondo la ricostruzione degli editori ottocenteschi (omessa, a ragion veduta, nelle più recenti edizioni di Perry e di Luzzatto-La Penna); cfr. più tardi Ignat. Diac. tetrast. 1, 7 Müller.

16. I due versi sono discussi molto bene dalla Albiani, art. cit. Sulla possibile paternità teocritea dell'epigramma vd. L. Rossi, The Epigrams Ascribed to Theocritus: A Method of Approach, LeuvenParis-Sterling, VA 2001, pp. 121-129.

17. È il n. 15 nella nuova edizione critica di F. Valerio (Venezia), che speriamo di vedere pubblicata in un futuro ormai prossimo. Le tre tipologie di variazione sono esaminate da J. Moore-Blunt, Enemies of the Vine: Three Epigrams, «Mnemosyne» n.s. 34, 1981, pp. 322-330.

18. A. Rosokoki, Die Erigone des Eratosthenes, Heidelberg 1995, in part. frr. 3-4 e pp. 81-86, con opportuni riferimenti ai classici studi di Maass, Solmsen e Merkelbach. Bibliografia più recente in D. Accorinti, Nonno di Panopoli. Le Dionisiache, IV (canti XL-XLVIII), Milano 2004, pp. 494-495. Notare la menzione del mito di Erigone, reinterpretato in chiave oscena, in Priap. 62.

19. Vd. il commento di Bömer a met. 15, 114-115 e a fast. 1, 354, con ampia documentazione e bibliografia.

20. Lo ha dimostrato con piena evidenza A.E. Housman, Mergere and Priap. 65, «CR» 29, 1915, pp. 173-174 (=Classical Papers III, Cambridge 1972, pp. 915-916). Cfr. di recente Jackson, art. cit., p. 259; Callebat, op. cit., pp. 262-263. Anche sul piano del significato mersit si impone, poiché il porcello, similmente a quanto avviene in Ovidio, non avrà divorato i fiori (cibo piuttosto improbabile): «the devastation spread by M. Grunnius Corocotta, solivertiator, when he and his snout get into the garden, consists much rather in routing up and in breaking down» (Housman, art. cit., p. $174=915$ ). 


\section{RIASSUNTI}

Il senso di Priapea 65 è una rivisitazione umoristica e dissacrante della tradizione poetica ellenistica (Eratostene, Leonida ed altri) ed augustea (in particolare Ovidio) che interpretava eziologicamente il sacrificio cruento di un animale come espiazione per il sacrilegio commesso da quest'ultimo e atto fondativo di una consuetudine religiosa.

INDICE

Mots-clés : Ératosthène, intertextualité, Léonidas de Tarente, Ovide, parodie, poésie hellénistique, Priapea, sacrifice 